\title{
Pericardial Release for Early LVAD Malalignment
}

\author{
Eric Jeng ${ }^{1}$, Alexander Ghannam² , and Mustafa Ahmed ${ }^{1}$ \\ ${ }^{1}$ University of Florida \\ ${ }^{2}$ University of Florida Health Science Center Jacksonville
}

April 27, 2020

\begin{abstract}
LS is a 39-year-old woman with systolic heart failure secondary to viral myocarditis (EF 10-15\%). She was transitioned from IABP support to LVAD. Five weeks postoperatively she experienced progressive low flow alarms and underwent pericardial release via left mini-thoracotomy. Flows immediately improved postoperatively.
\end{abstract}

Pericardial Release for Early LVAD MalalignmentAuthor Block: E. I. Jeng ${ }^{1}$, A. D. Ghannam² ${ }^{2}$ M. M. Ahmed ${ }^{3}$,

${ }^{1}$ Surgery, University of Florida, Gainesville, FL, ${ }^{2}$ Surgery, University of Florida, Jacksonville, FL, ${ }^{3}$ Medicine, University of Florida, Gainesville, FL,

Introduction: Cardiogenic shock is a state of low cardiac output resulting in life threatening end organ hypoperfusion and hypoxia. ${ }^{1}$ Mechanical circulatory support is often a necessary therapy for those who fail medical management. ${ }^{2,3}$ While durable LVAD is a sustainable option for many patients, ${ }^{4,5}$ the pitfalls of these therapies should be described to offer solutions to potentially recurring problems. Only through optimizing medical and surgical management options, can we continue to improve the lives of the patients on the continuum of cardiogenic shock and end-stage heart failure.

Methods: Pericardial release through a left mini anterior thoracotomy.

Results: LS is a 39-year-old woman with a medical history significant for systolic heart failure secondary to viral myocarditis. She was medically managed by the heart failure team for over a year, and her heart function stabilized with left ventricular ejection fraction (LVEF) greater than $40 \%$. When she became pregnant in 2018, her LVEF declined to 10-15\%. Six months after an uneventful cesarean section she presented in ambulatory cardiogenic shock, with a $40 \mathrm{lb}$ weight gain, jugular venous distention to the level of the mandible, and acute renal injury.

She was started on inotropes, underwent aggressive diuresis, and had an axillary intra-aortic balloon pump (IABP) placed. LS was discussed at the Medical Review Board and listed for orthotropic heart transplant (OHTx). Despite two weeks of ambulatory IABP support, LS progressively deteriorated, and so we elected to bridge-to-transplant with a pericardial placed Heartware (Medtronic, Minneapolis, MN) LVAD via sternotomy.

LS did well post-operatively with flows averaging 4-5 L/min. However, over the next four weeks, her LVAD intermittently experienced low flow alarms to $2.5 \mathrm{~L} / \mathrm{min}$ with MAPs greater than $90 \mathrm{mmHg}$. TTE evaluation demonstrated a midline septum and the aortic valve opening with every beat, so the RPMs were increased in addition to continue afterload reduction. Despite these interventions, LS had progressively worsening low flow alarms, and was now flowing consistently at $1.5 \mathrm{~L} / \mathrm{min}$ (Figure 1). On postoperative day 34, we elected for surgical reintervention. We proceeded with a left mini anterior thoracotomy for pericardial release. With 
the pericardium exposed, an incision in the pericardium was made superior and parallel to the phrenic nerve $3 \mathrm{~cm}$ cranial and caudal to the LVAD. It was clear that the LVAD had been displaced in the pericardium superiorly and anteriorly with the inflow cannula now directed at the septum. By reangling the LVAD inflow, pump flows immediately improved to $>4.5 \mathrm{liter} / \mathrm{min}$. Two 0 -prolenes were used to anchor the pump to the chest wall (Figure 2).

Postoperatively, LS experienced normalization of her right atrial, pulmonary artery and wedge pressures. She recovered well following the pericardial release. Nine months later, she underwent an uneventful OHTx and has been discharged home.

Conclusion: The LVAD device pocket can undergo contraction in the postoperative period changing the angle of the inflow or outflow cannula. ${ }^{6}$ This has been described previously in pre-peritoneal placed LVADs, but is not well known in pericardial placed LVADs. In patient LS, we noticed a steady decline in LVAD flows over a five week interval due to a progressive device migration. In order to optimize end-organ support and to prevent pump thrombosis we employed a pericardial release and device anchor along the chest wall. We were able to utilize this as a durable solution for LVAD malalignment, and successfully bridge this patient to OHTx.

\section{Author Contributions:}

Eric I. Jeng provided the oversight of the project and critical revision of article. Alexander D. Ghannam drafted the article. Mustafa M. Ahmed revised and approved the article.

Key Clinical Message: A previously functioning LVAD that experiences low flow alarms may benefit from minimally invasive pericardial release, rather than LVAD replacement, to improve inflow and outflow cannula angles and ultimately hemodynamics.

\section{Resources:}

1. Chaudhry, S. P., \& Stewart, G. C. (2016). Advanced Heart Failure: Prevalence, Natural History, and Prognosis. Heart Failure Clinics, 12 (3), 323-333.

2. Diepen, S. V., Katz, J. N., Albert, N. M., Henry, T. D., Jacobs, A. K., Kapur, N. K., .. Cohen, M. G. (2017). Contemporary Management of Cardiogenic Shock: A Scientific Statement From the American Heart Association. Circulation, 136 (16).

3. Eisen, H. J. (2019). Left Ventricular Assist Devices (LVADS): History, Clinical Application and Complications. Korean Circulation Journal , 49 (7), 568. doi: 10.4070/kcj.2019.0161

4. Kilic, A., Acker, M., \& Atluri, P. (2015). Dealing with surgical left ventricular assist device complications. Journal of Thoracic Disease, 7 (12), 2158-2164.

5. Hunt, S. A., \& Ea, R. (2002). The REMATCH trial: Long-term use of a left ventricular assist device for end-stage heart failure. Journal of Cardiac Failure , 8 (2), 59-60. doi: 10.1054/jcaf.2002.32944

6. Adamson, RM., et al. "Principles of HeartMate II Implantation to Avoid Pump Malposition and Migration." Journal of Cardiac Surgery, vol. 30, no. 3, Apr. 2014, pp. 296-299., doi:10.1111/jocs.12478. 


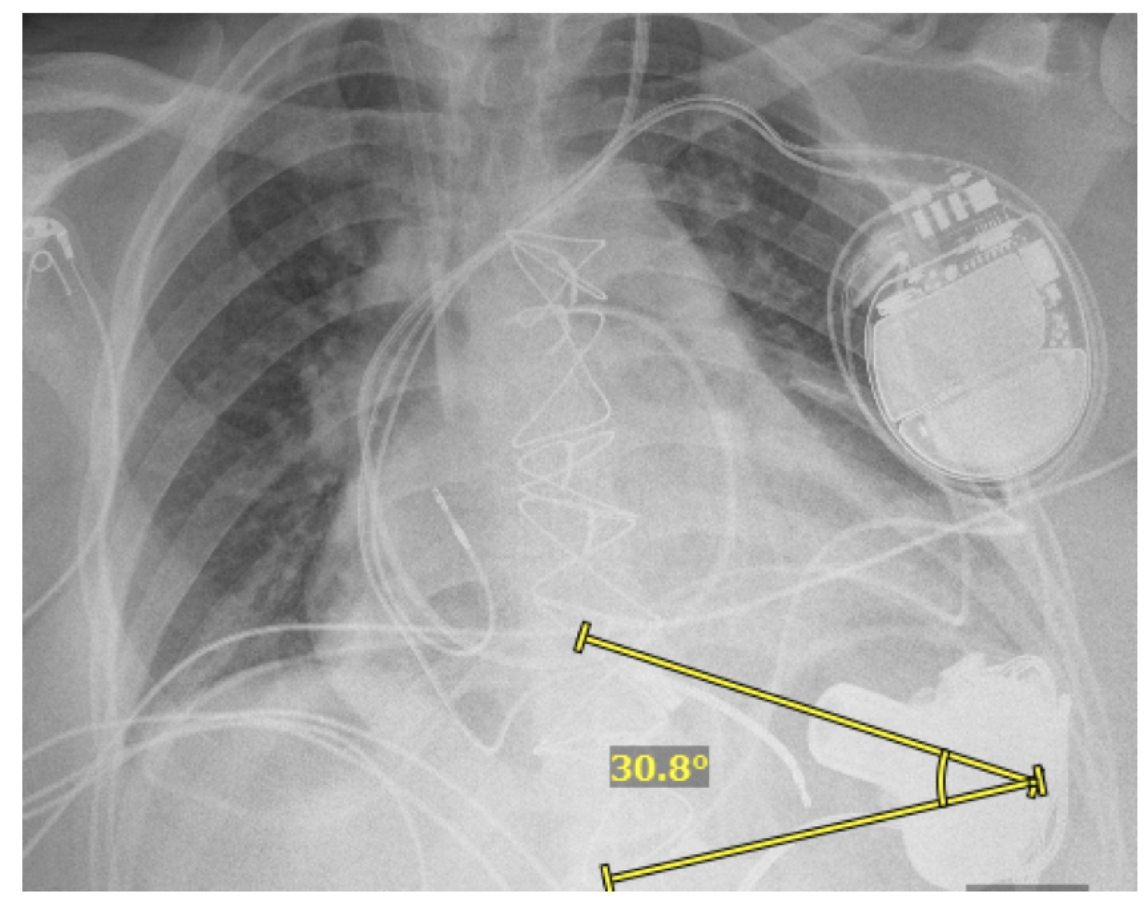

Figure 1. Low Flow 


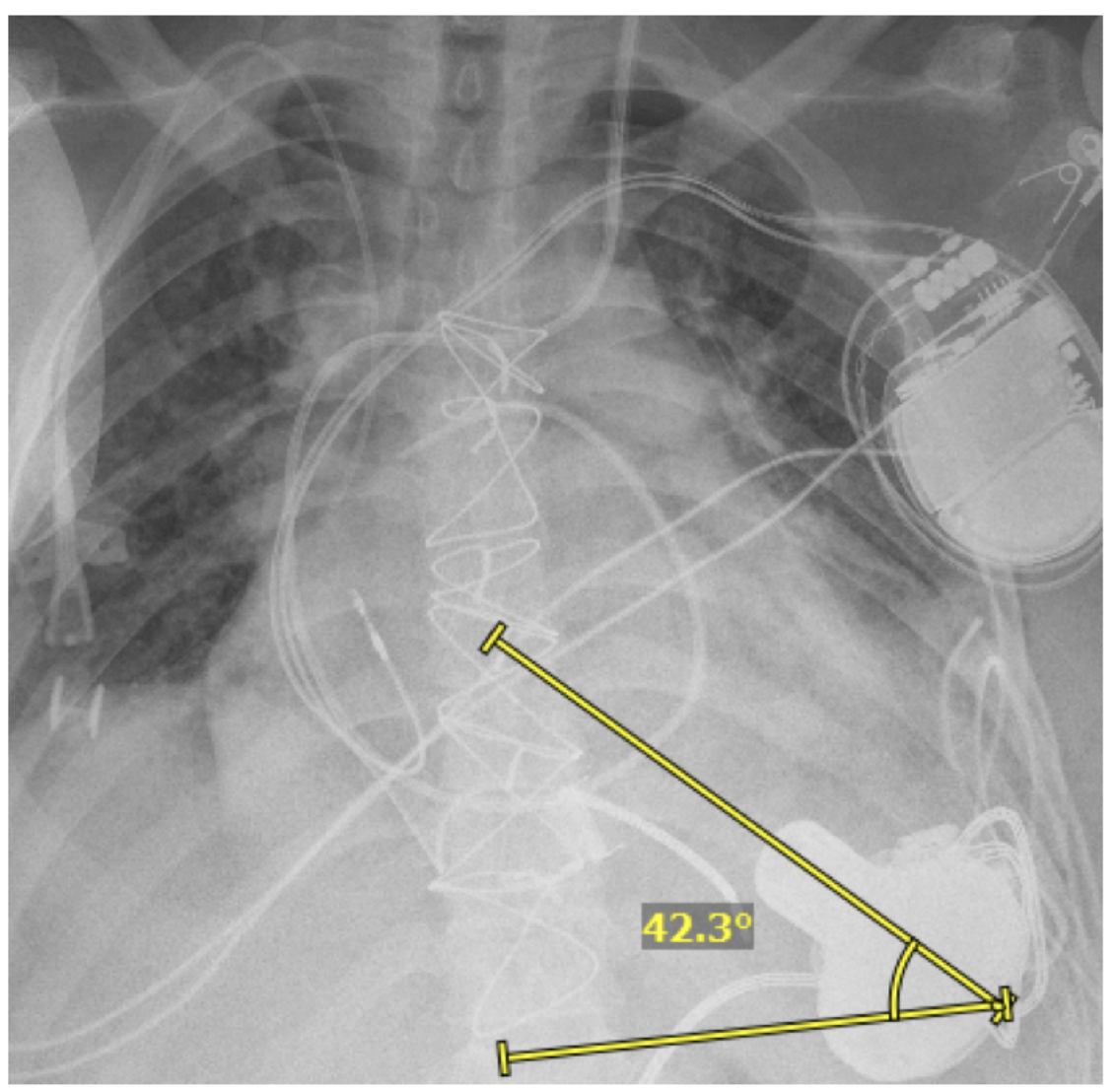

Figure 2. Pericardial Release 